\title{
Estudo da sinterização de uma liga Fe-22,5Cr-5,5Ni obtida pela mistura de pós elementares
}

\author{
Study of sintering of a Fe-22,5Cr-5,5Ni alloy \\ obtained by elemental powders mixture
}

Ederson Bitencourt das Neves ${ }^{1}$, Luciano Volcanoglo Biehl ${ }^{2}$ Alice Gonçalves Osorio ${ }^{3}$, Tarique Hernandez Schneider ${ }^{4}$

\author{
1,2,4 Escola de Engenharia/ FURG- Av. Itália, km 8, Campus Carreiros 96203-900, Rio Grande, RS. \\ e-mail: ppmec.secretaria@furg.br \\ ${ }^{3}$ Centro de Desenvolvimento Tecnológico - CDTec, UFPel- Universidade Federal de Pelotas \\ e-mail: edersonbn@gmail.com ${ }^{1}$ lucianobiehl@gmail.com² osorio.alice@gmail.com³ \\ tarique_hernandez_schneider@hotmail.com ${ }^{4}$
}

\section{RESUMO}

Usualmente a sinterização de Aços Inoxidáveis AID (ligas Fe-Cr-Ni) ocorre a partir de pós pré-ligados de AID ou pelo balanceamento entre pós de aços inoxidáveis austeníticos misturado com pós de aços inoxidáveis ferríticos, proporcionando uma microestrutura duplex. O objetivo deste trabalho foi investigar a possibilidade de desenvolver uma rota alternativa de produção do AID com menor custo, além de avaliar as características microestruturais e mecânicas da liga Fe-22,5Cr-5,5Ni. Neste contexto, buscou-se desenvolver uma liga a partir de misturas de pós elementares de formulação similar ao AID UNS S32205 e com diferentes isotermas buscando a difusão entre os elementos, com o intuito de eliminar a necessidade de obtenção de pós pré-ligados de elevado custo. Os pós de partida foram caracterizados por Difração de Raios X (DRX) e Microscopia Eletrônica de Varredura (MEV), e empregados na produção de 10 (dez) formulações que foram uniaxialmente conformadas em pastilhas de 18,88 mm de diâmetro e tratadas termicamente com tempos variando entre 1 (uma) e 6 (seis) horas; com isotermas de $1000^{\circ} \mathrm{C} ; 1100^{\circ} \mathrm{C}$ e $1200^{\circ} \mathrm{C}$ e sob atmosfera controlada de nitrogênio. Com o uso de Microscopia Óptica (MO), foram selecionadas 4 (quatro) misturas para avaliação complementar. Esse estudo englobou a análise de difusão dos elementos Fe, Cr e Ni por Espectroscopia de Energia Dispersiva (EDS) acoplada ao MEV, e avaliação microestrutural por (MO, MEV, DRX). Os resultados obtidos com as análises por MO e MEV revelaram uma baixa difusão do cromo na matriz de ferro. Espectros obtidos por DRX apresentaram a formação de picos de ferrita e em algumas das amostras pequenos picos de austenita, não sendo possível apontar a formação de uma estrutura duplex. A análise de espectroscopia Raman indicou a formação de óxido de cromo no entorno das partículas de cromo, evitando sua difusão na matriz ferrítica. Por fim, os ensaios de microdureza revelaram uma relação diretamente proporcional ao aumento da temperatura e tempo de sinterização com as microdurezas apresentadas pelas misturas obtidas com pós elementares.

Palavras-chave: Misturas de pós metálicos. Aço inoxidável duplex. Sinterização de ligas Fe-Cr-Ni.

\section{ABSTRACT}

Usually the Sintering of Duplex Stainless Steels SS (Fe-Cr-Ni alloy) from prebounded powders of duplex stainless steels or a balance mixture between austenitic and ferritic powders are used to form a duplex microstructure. The objective of this work was to investigate the possibility of developing an alternative route to produce Duplex SS in a rather low cost, and, in addition, to evaluate the microstructural and mechanical characteristics of the Fe-22,5Cr-5,5Ni alloy. In this context, it was pursued the development of an alloy by the mixing of elementary powders with similar formulation of the duplex SS UNS S32205, in order to eliminate the need of obtaining prebounded powders. The powders were characterized by X-ray Diffraction (XRD) and Scanning Electron Microscopy (SEM) to be used in the production of 10 (ten) uniaxial tablet shaped mixtures of $18,88 \mathrm{~mm}$ of diameter and thermally treated varying times between 1 (one) to 6 (six) hours; isotherms from $1000^{\circ} \mathrm{C}\left(1273 \mathrm{~K} / 1832^{\circ} \mathrm{F}\right) ; 1100^{\circ} \mathrm{C}\left(1373 \mathrm{~K} / 2012^{\circ} \mathrm{F}\right)$ and $1200^{\circ} \mathrm{C}\left(1473 \mathrm{~K} / 2192^{\circ} \mathrm{F}\right)$ and 
under nitrogen controlled atmosphere. Optical Microscopy (OM) allow one to selected 4 (four) sintered mixtures to further evaluation. This study comprises the diffusion analysis of $\mathrm{Fe}$, $\mathrm{Cr}$ and Ni elements using SEM by Energy Dispersive Spectrometer (EDS) and microstructural evaluation using (OM, SEM, XRD). The results obtained from OM and SEM analysis revealed the low diffusivity of chrome in the iron matrix. XRD spectra showed peaks of ferrite formation and austenite in some of the samples, so it was not possible to indicate the formation of a duplex structure. The Raman spectra indicated chromium oxide in the vicinity of chromium particles, preventing its spread to the ferritic matrix. Finally, the microhardness tests revealed a direct proportion to the increase of temperature and sintering time with the microhardness submitted by the mixtures obtained with elemental powders.

Keywords: Mixing metal powders. Duplex Stainless Steel. Sintering of alloys Fe-Cr-Ni

\section{INTRODUÇÃO}

Os Aços Inoxidáveis Duplex (AID) fazem parte de uma linha especial de materiais que vem despertando o interesse de pesquisadores, fabricantes e usuários. Os mesmos tratam-se de ligas Fe-Cr-Ni que contêm até 0,30\% em peso de nitrogênio na forma atômica e apresentam microestruturas bifásicas compostas por uma matriz ferrítica (C.C.C.) com ilhas de austenita (C.F.C.), precipitada com morfologia arredondada e alongada. Esses materiais tem aplicação na produção de componentes de equipamentos de perfuração, corpos de válvulas e tubulações utilizadas principalmente em indústrias petroquímicas e offshore. O seu emprego está relacionado à sua excelente resistência em ambientes corrosivos, bem como a sua alta resistência mecânica aliada a uma boa tenacidade [1,2,3].

As ligas AID podem ser obtidas por misturas de pós ferríticos, pós austeníticos ou martensíticos com pós-elementares, provocando a sua desestabilização e produzindo microestruturas bifásicas ou também pelo balanceamento entre pós de aços inoxidáveis austeníticos misturado com pós de aços inoxidáveis ferríticos proporcionando uma microestrutura duplex (austenita-ferrita). O autor Moinhos [4], obteve um aço inoxidável duplex a partir da mistura de pós elementares, com o emprego do processo de moagem de alta energia em um moinho do tipo Attritor, $\mathrm{O}$ moinho de alta energia proporcionou a soldagem a frio das partículas por ação do efeito mecânico, agregando os fragmentos/pós e facilitando a difusão entre os elementos presentes da mistura. Outras pesquisas que utilizaram a Metalurgia do Pó (M/P) para obtenção de AID, optaram pelo uso de pós pré-ligados. Nestas pesquisas pós inoxidáveis austeníticos foram adicionados a pós inoxidáveis ferríticos, o que garanti uma boa difusividade entre os elementos constituintes (DOBRZANSKI et al. [5]; GARCIA et al. [6]; MARTIN et al.[7]; e TANSKI et al. [8]),

Com base nestes estudos, este trabalho teve por objetivo principal investigar a sinterização dos elementos em uma liga Fe-22,5Cr-5,5Ni obtida a partir da M/P, composição esta, característica de AID. Adicionalmente, as características microestruturais e mecânicas desta liga foram avaliadas. A liga em estudo foi obtida por uma rota alternativa de sinterização, que envolveu a mistura de pós elementares comerciais, sem uso de moagem de alta energia e utilizando como referência de composição química, uma formulação similar ao aço inoxidável duplex UNS S32205. Para isso, foram realizadas: avaliação da difusividade dos elementos de liga na matriz de ferro pela técnica de Espectroscopia de Energia Dispersiva (EDS); verificação de formação das fases austenita e ferrita nas misturas por DRX e análise de microdureza Vickers.

\section{MATERIAIS E MÉTODO}

Para o processo de sinterização dos pós elementares dissociados, a composição química utilizada como padrão foi a do aço AID UNS S32205, conforme formulações apresentadas na Tabela 1.

Tabela 1: Composições em (\%) do AID UNS S32205 [9] e das misturas utilizadas no trabalho

\begin{tabular}{|c|c|c|c|c|c|c|c|c|c|c|}
\hline \multicolumn{11}{|c|}{ Elemento (\%) } \\
\hline & C & $M n$ & Si & $\mathrm{Cr}$ & $\mathrm{Ni}$ & $\mathbf{P}$ & $S$ & Mo & $\mathrm{N}$ & $\mathrm{Fe}$ \\
\hline $\begin{array}{c}\text { Aço UNS } \\
\text { S32205 }\end{array}$ & 0,03 & 2,00 & 1,00 & $\begin{array}{l}22,0 \\
23,0\end{array}$ & $\begin{array}{l}4,50 \\
6,50\end{array}$ & 0,03 & 0,02 & $\begin{array}{l}3,0 \\
3,5\end{array}$ & $\begin{array}{l}0,14 \\
0,20\end{array}$ & - \\
\hline $\begin{array}{l}\text { Composição } \\
\text { das Amostras }\end{array}$ & 0,03 & 2,00 & 1,00 & 22,5 & 5,5 & 0,03 & 0,02 & 3,5 & 0,2 & - \\
\hline
\end{tabular}

A Tabela 2 apresenta as características de pureza e os fornecedores dos materiais utilizados no desenvolvimento deste trabalho. 
NEVES, E. B.; BIEHL, L.V.; OSORIO, A. G.; SCHNEIDER, T. H. revista Matéria, v.21, n.1, pp. 185 - 195.2016.

Tabela 2: Características dos pós comerciais e o gás Nitrogênio utilizado.

\begin{tabular}{|c|c|c|}
\hline ELEMENTO & \% (pureza) & Fornecedor \\
\hline $\begin{array}{l}\text { Fe com lubrificante } \\
\text { Acrawax }{ }^{\circledR} \text { com } 1 \% \\
\text { em peso. }\end{array}$ & $>99$ & $\begin{array}{l}\text { BRATS Indústria e Comercio de Produtos Metálicos } \\
\text { Especiais Ltda. }\end{array}$ \\
\hline Cr e Ni & $>99$ & CITRA do Brasil Comércio Internacional Ltda. \\
\hline Mn & $>63$ & Microquímica Ind. Químicas Ltda \\
\hline Mo & $>66$ & Microquímica Ind. Químicas Ltda \\
\hline $\mathrm{N}_{2}$ & $>99$ & White Martins Praxair Inc. \\
\hline
\end{tabular}

As formulações a serem estudadas foram processadas em um misturador do tipo "Y” a 45 r.p.m. durante 120 minutos. A etapa seguinte consistiu na pesagem de $10 \mathrm{~g}$ do pó para posterior compactação. Para a compactação das amostras foi utilizada uma matriz uniaxial de duplo efeito com pressão de compactação de $800 \mathrm{MPa}$ formando pastilhas com diâmetro e altura média de $\varnothing=18,88$ e h=5,5mm respectivamente. A densidade a verde obtida para as amostras foi de $4,42 \mathrm{~g} / \mathrm{cm}^{3}$.

Para sinterização foram realizados tratamentos térmicos de solubilização com isoterma de $1000^{\circ} \mathrm{C}$, $1100^{\circ} \mathrm{C}$ e $1200^{\circ} \mathrm{C}$ em forno mufla EDG Série Inox Line até $1200^{\circ} \mathrm{C}$ com atmosfera controlada. O uso destas isotermas em conjunto com a etapa de resfriamento rápido teve por finalidade dissolver os precipitados indesejados na estrutura do aço inoxidável duplex. Os parâmetros de sinterização utilizados nas misturas podem ser visualizados na Tabela 3, foram produzidas 10 misturas e sinterizadas em atmosfera controlada em nitrogênio, com taxa de aquecimento de $5{ }^{\circ} \mathrm{C} /$ minuto. Os tempos registrados de 60, 120, 180 e 360 minutos foram contados a partir do momento em que as temperaturas de $1000^{\circ} \mathrm{C}, 1100^{\circ} \mathrm{C}$ e $1200^{\circ} \mathrm{C}$ eram alcançadas.

Tabela 3: Parâmetros de tempo e temperatura utilizados na sinterização das formulações.

\begin{tabular}{ccc}
\hline $\begin{array}{c}\text { Mistura } \\
\text { (formulações) }\end{array}$ & $\begin{array}{c}\text { Tempo } \\
\text { (Minutos) }\end{array}$ & Isoterma $\left({ }^{\circ} \mathbf{C}\right)$ \\
\hline M1 & 60 & 1000 \\
M2 & 120 & 1000 \\
M3 & 180 & 1000 \\
M4 & 60 & 1100 \\
M5 & 120 & 1100 \\
M6 & 180 & 1100 \\
M7 & 60 & 1200 \\
M8 & 120 & 1200 \\
M9 & 180 & 1200 \\
M10 & 360 & 1200 \\
\hline
\end{tabular}

Após a sinterização, as amostras passaram pelo processo de preparação metalográfica, onde as amostras em forma de pastilha foram cortadas as pastilhas em 2 partes iguais empregando uma cortadeira metalográfica (marca: BUHLER, modelo: Delta Abrasimet). A seguir, foram embutidas a quente com baquelite em uma embutidora manual (marca: BUHLER, modelo: Simplimet 2). Para o lixamento foram utilizadas lixas d’água com granulometria de 400, 600, 1000 e 1200 mesh e para o polimento com politriz semi-automática com alumina como agente de polimento (marca: STRUERS).

Para análise microestrutural foi usado um microscópio óptico de luz refletida com sistema de análise de imagens - (Olympus GX 51S com Sistema de aquisição de imagens digital). As análises por difração de raios X foram realizadas em um Difratômetro de raios X, marca Rigaku (modelo Ultima IV). O tubo usado foi o de Cu (radiação Kalfa - $\lambda=1,54059$ Â), com passo de $0,02^{\circ}$ e tempo de integração de 1 segundo por ponto, no intervalo de $30^{\circ}$ a $100^{\circ}$ em $2(\theta)$. Os difratogramas obtidos foram analisados e identificados pela comparação com as fichas de padrões do "International Centre for Diffraction Data (ICDD)".

Ainda na análise microestrutural, foi necessária a utilização de um microscópio eletrônico de varredu- 
ra (MEV), os mapeamentos por MEV foram realizados no equipamento da marca JEOL modelo JSM 6610L (MEV de alto e baixo vácuo) e equipado com JEOL JSM 6610LV Detectores de EDS. Foram realizadas em cada amostra 40 (quarenta) varreduras (framers) com uma distância de trabalho igual a $10 \mathrm{~mm}$. As análises de microdureza Vickers foram realizadas com o Microdurômetro da marca Shimadzu e modelo HMV-2T e os ensaios foram conduzidos empregando uma carga de $(0,5 \mathrm{kgf})$ e tempo de aplicação de 10 s.Um espectrômetro da marca Renishaw inVia foi utilizado para a avaliação das partículas de cromo. O laser utilizado foi de $532 \mu \mathrm{m}$ de comprimento de onda. Foram realizadas três medidas para cada amostra analisada.

\section{RESULTADOS E DISCUSSÕES}

A partir das imagens obtidas em microscopia óptica (MO) para cada uma das 10 amostras produzidas em diferentes temperaturas e tempo de isoterma (vide Tabela 2), avaliou-se que 4 destas amostras podem ser utilizadas para representar todas as misturas produzidas, uma vez que entre elas não se observou significativa mudança microestrutural. A seguir, as 4 amostras foram separadas para análises complementares descritas abaixo.

\subsection{ANÁLISE POR MICROSCOPIA ÓPTICA}

A Figura 1 apresenta imagens capturadas no MO com as 4 misturas M1, M5, M8 e M10 selecionadas para análise complementar.

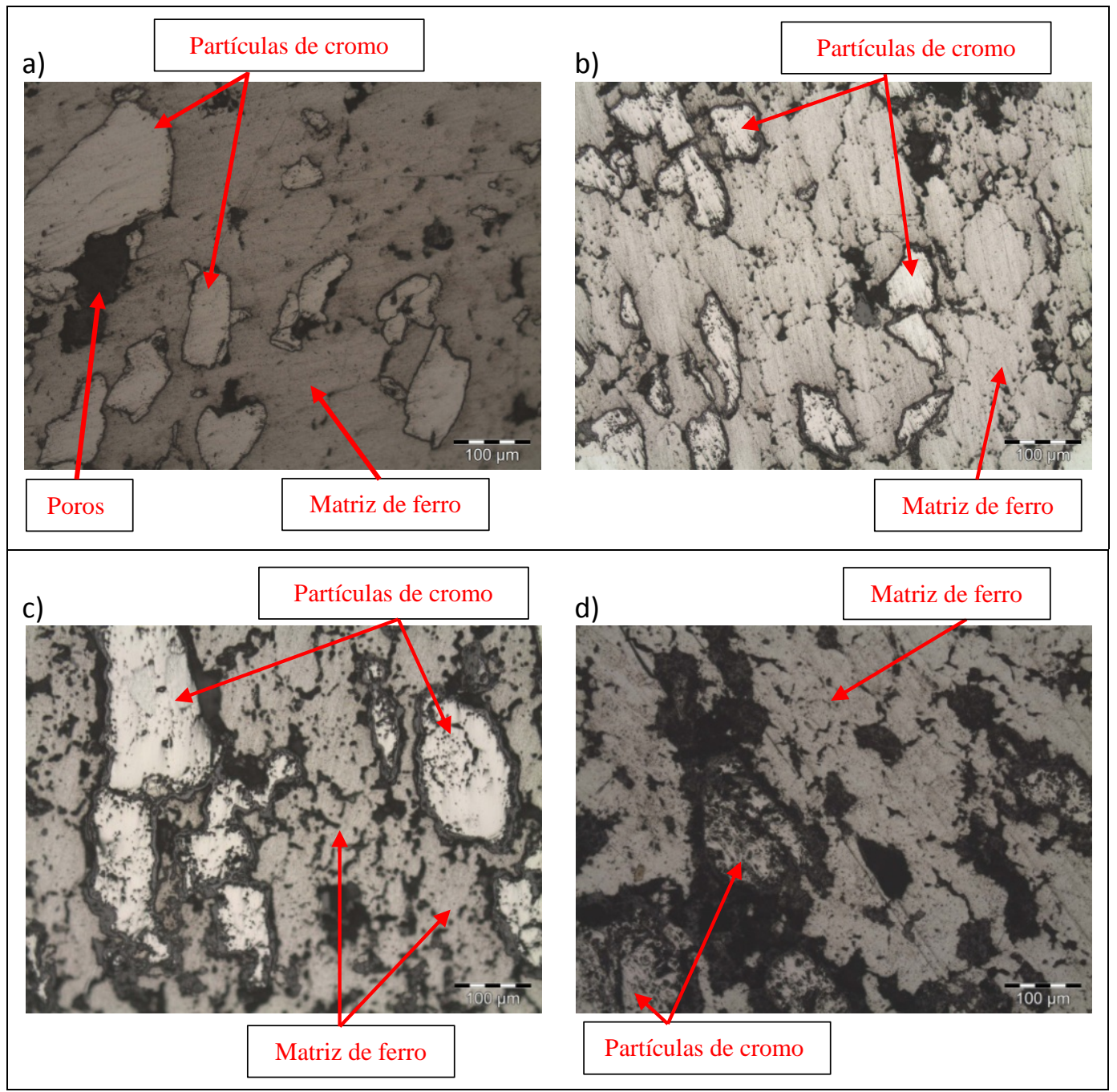

Figura 1: Microscopia ótica das misturas selecionadas. a) $\mathrm{M} 1-1000^{\circ} \mathrm{C} / 60$ minutos b) $\mathrm{M} 5-1100^{\circ} \mathrm{C} / 120$ minutos c) M8$1200^{\circ} \mathrm{C} / 120$ minutos d) $\mathrm{M} 10-1200^{\circ} \mathrm{C} / 360$ minutos

As imagens de MO (Figura 1) indicam uma difusão incompleta para algumas partículas das misturas. No entanto verifica-se nas Figuras 1b, 1c e 1d um aumento da difusão no entorno destas partículas, que pode ser atribuído a elevação da temperatura de sinterização, bem como do tempo de permanência das misturas no 
forno (Figura 1d). Apesar de bastante informativa, as análises de MO não indicam quais partículas não difundiram na matriz, sendo assim, análises de MEV/EDS foram necessárias para identificar as mesmas.

\subsection{ANÁLISE POR DIFRAÇÃO DE RAIOS X}

A caracterização por DRX foi utilizada para identificar as fases austenita (C.F.C) e ferrita (C.C.C). Essa particularidade viabilizada por esta técnica, faz com que o mesma seja largamente utilizada, principalmente nos casos em que deseja-se identificar duas fases de mesma composição, porém com diferentes estruturas cristalinas [10]. A Figura 2 apresenta os difratogramas para as misturas M1, M5, M8 e M10.

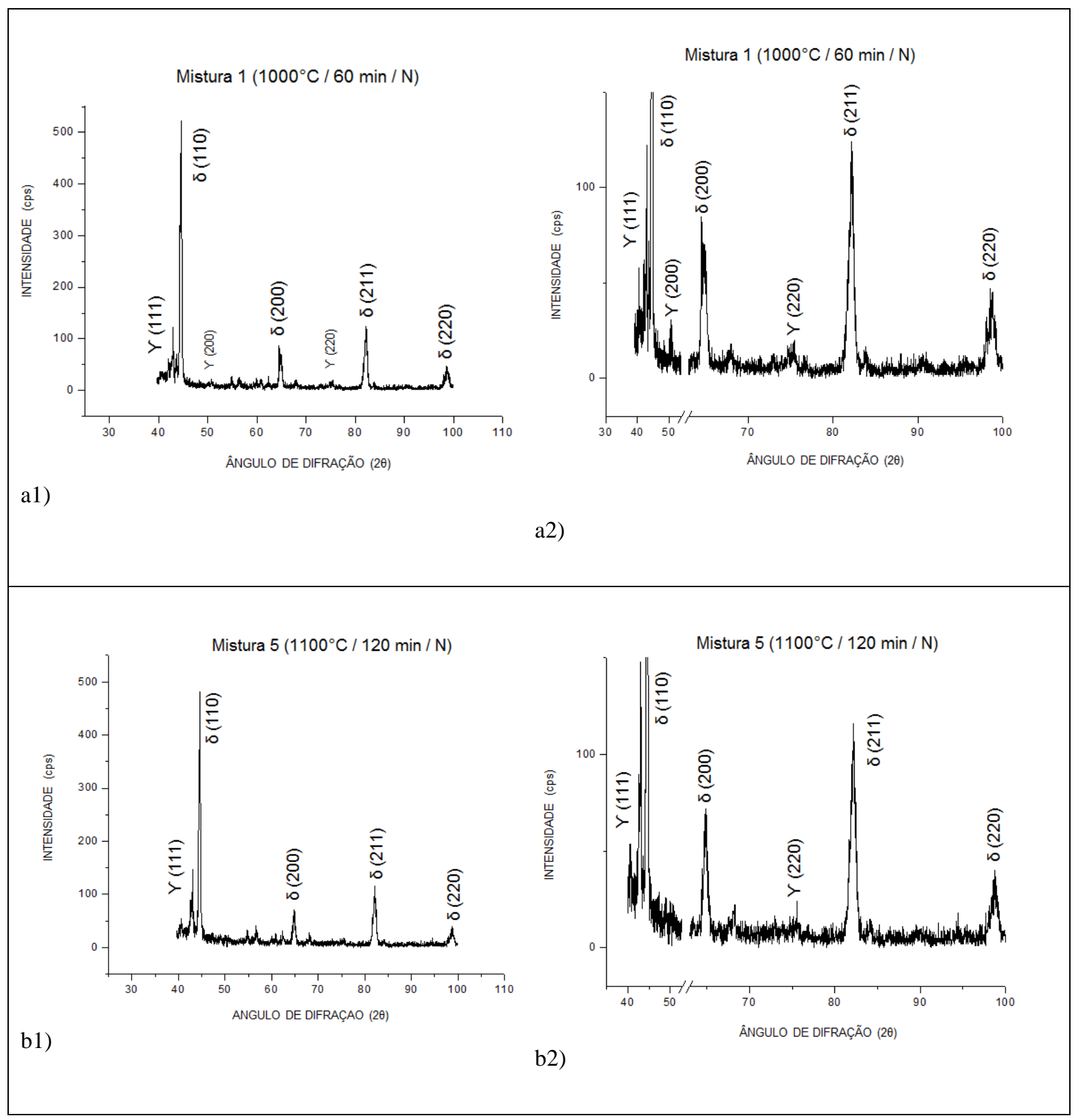




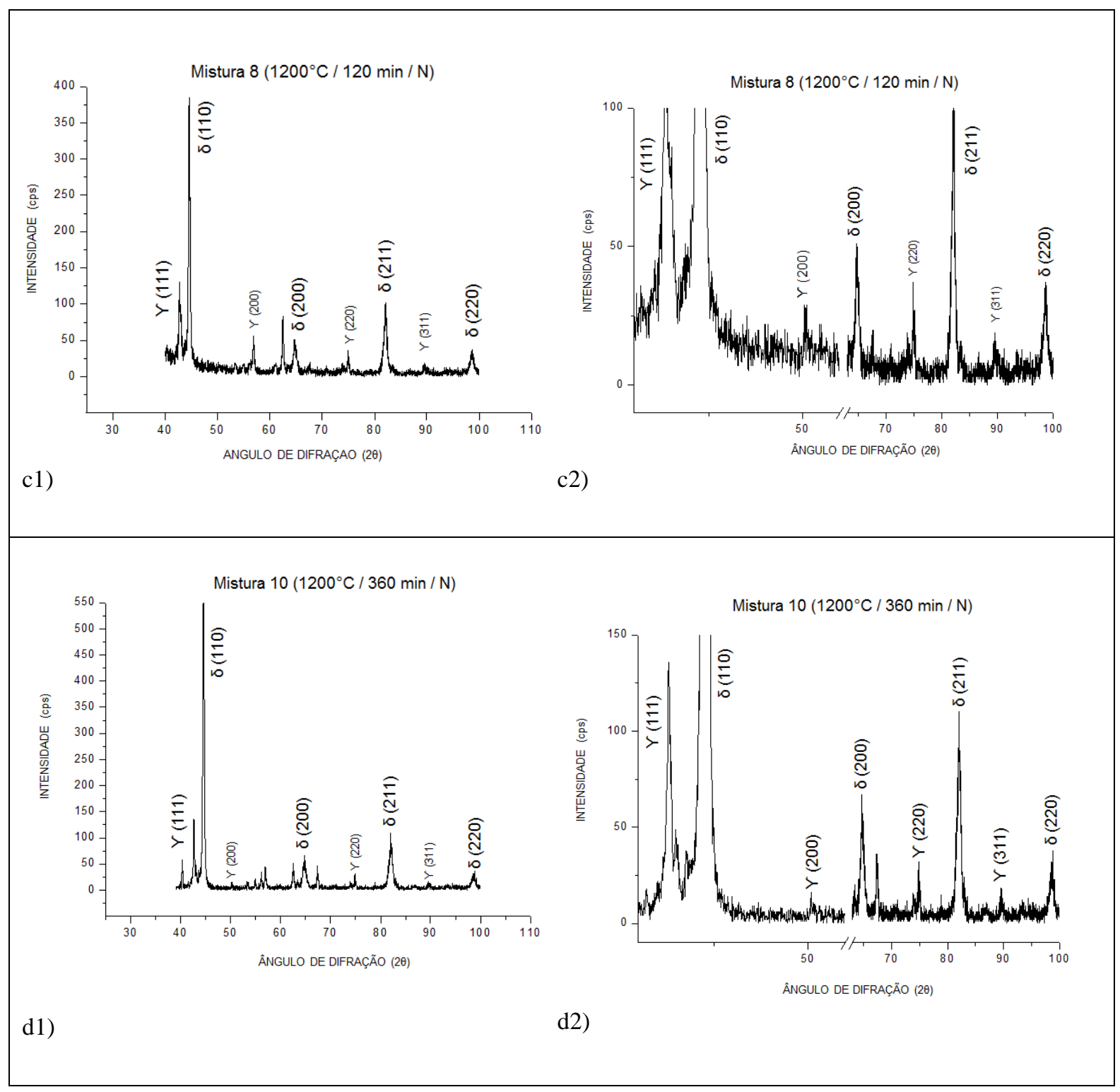

Figura 2: Difratogramas de raios $\mathrm{X}$ das misturas: a1) $\mathrm{M} 1\left(1000^{\circ} \mathrm{C} / 60\right.$ minutos $)$ e a2) $\mathrm{M} 1\left(1000^{\circ} \mathrm{C} / 60\right.$ minutos $)$ com ampliação de escala nos eixos " $x$ ” e " $y$ ”, b1) M5( $1100^{\circ} \mathrm{C} / 120$ minutos) e b2)M5(1100 $\mathrm{C} / 120$ minutos) com ampliação de escala nos eixos " $x$ " e "y", c1) M8(1200 ${ }^{\circ} \mathrm{C} / 120$ minutos $)$ e c2) $\mathrm{M} 8\left(1200^{\circ} \mathrm{C} / 120\right.$ minutos $)$ com ampliação de escala nos eixos "x" e “y”, d1) M10 (1200C/360 minutos) e d2) M10 (1200C/360 minutos) com ampliação de escala nos eixos "x" e "y"

Ao analisar a Figura 2 se verifica as fases presentes conforme os cartões de referência ICDD (00-0330397) para austenita ( $\Upsilon$ ) e ICDD (00-054-0331) para ferrita ( $\delta$ ) [11]. Esta análise evidencia a formação das fases características de uma estrutura duplex no aço desenvolvido.

\subsection{ANÁLISE POR ESPECTROSCOPIA RAMAN}

Foi realizada uma análise de espectroscopia Micro-Raman nas amostras sinterizadas para verificar a possibilidade das partículas de cromo terem reagido e formado compostos não-metálicos.

Os resultados obtidos a partir desta técnica foram muito simulares para todas as amostras, sendo assim, apenas alguns espectros são apresentados na Figura 3. A análise foi realizada na matriz na interface entre a matriz e Cr e no núcleo da partícula de Cr.

As análises realizadas na matriz e no núcleo da partícula de Cr não geraram sinal, o que é esperado para componentes metálicos, que não apresentam efeito Raman significativo. Já na interface entre a matriz e a partícula não difundida, se observou o sinal mostrado na Figura 3. Neste espectro se observa uma banda de maior intensidade na frequência de aproximadamente $652 \mathrm{~cm}^{-1}$. 
Segundo literatura consultada a banda característica de óxido de cromo (III) é verificada em valores um pouco menores que este [12], no entanto, o espectro obtido apresenta o mesmo padrão de picos do óxido de cromo, porém com um deslocamento para valores maiores.

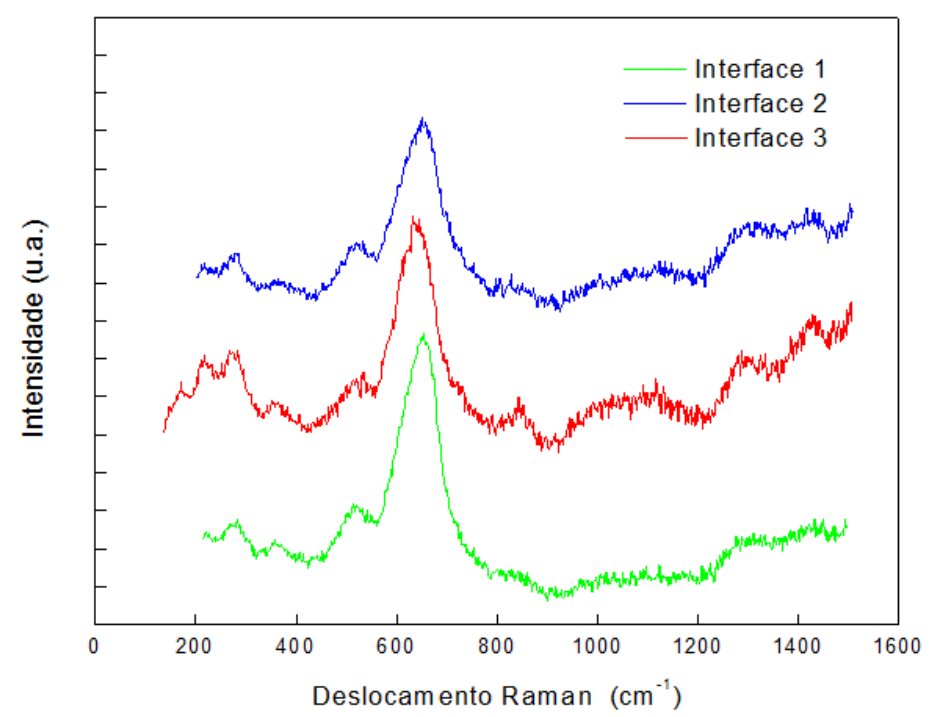

Figura 3: Espectros micro-Raman na interface localizada entre a matriz de ferro e a partícula de Cr das amostras M1(azul), M5(vermelho) e M8(verde).

Este deslocamento se atribui a uma possível falta de alinhamento do feixe de laser ou deformação plástica da microestrutura. Desta forma, fica evidenciada a formação de uma camada óxida ao redor das partículas de cromo, o que pode ter impedido a difusão do Cr na matriz de ferro.

\subsection{ANÁLISE POR MICROSCOPIA ELETRÔNICA DE VARREDURA}

Existe uma grande dificuldade na detecção da difusividade de elementos metálicos dentro de matrizes de ferro. Uma forma indireta de compreender esta difusão é através da técnica que se utiliza de um filtro por elementos, baseando no funcionamento do EDS, e se projeta o resultado na forma de imagem com cores distintas [4, 13]. Nas micrografias apresentadas na Figura 4, pode-se visualizar a imagem capturada pelo MEV das misturas com tratamento térmico em atmosfera de nitrogênio, junto com o mapeamento realizado pelo EDS, que evidencia indícios de difusão dos elementos na mistura. 


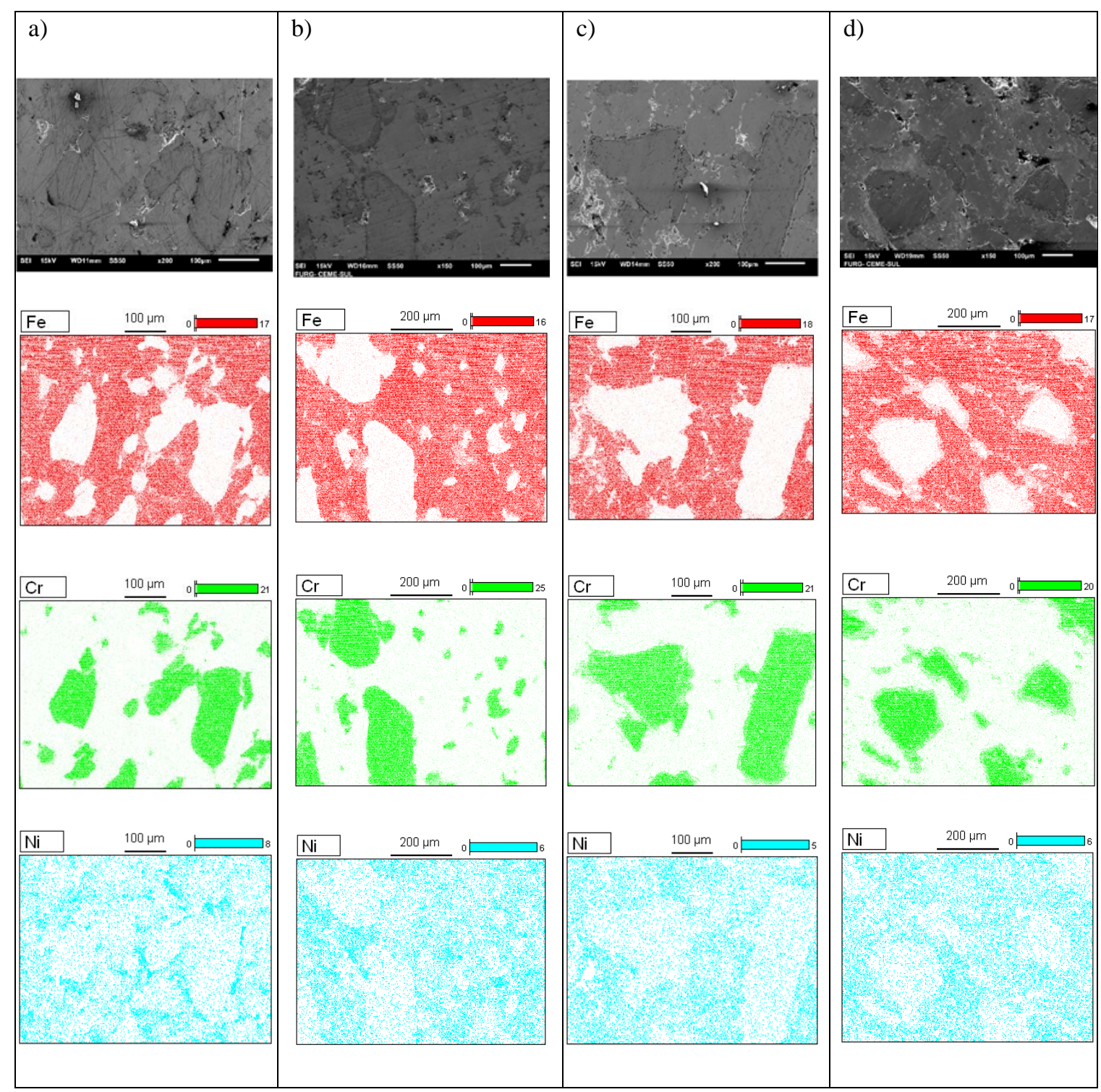

Figura 4: a) Micrografia de varredura das misturas com mapeamentos por EDS com cores em vermelho para o ferro, de verde para o cromo e azul para o níquel. Aumentos em função das barras de escalas das imagens: a) $\mathrm{M} 1\left(1000^{\circ} \mathrm{C} / 60 \mathrm{minu}-\right.$ tos), b) M5( $1100^{\circ} \mathrm{C} / 120$ minutos), c) $\mathrm{M} 8\left(1200^{\circ} \mathrm{C} / 120\right.$ minutos $)$, d) $\mathrm{M} 10$ ( $\left(1200^{\circ} \mathrm{C} / 360\right.$ minutos $)$

Na Figura 4 aplicando o filtro EDS se observa que o Ni (em azul) apresentou boa difusão na matriz de Fe (em vermelho) e nas partículas de Cr, porém em menor proporção do que a apresentada na matriz de Fe, sendo possível inclusive observar os contornos da interface Fe-Cr nos filtros de Ni. No entanto, o mesmo não pode ser dito das partículas de $\mathrm{Cr}$ (em verde), que não apresentaram boa difusão na matriz de Fe. 


\subsection{ENSAIOS DE MICRODUREZA VICKERS}

Os ensaios de microdureza HV0,5 foram realizados para todas as amostras obtidas a partir das misturas acima mencionadas, permitindo determinar a distribuição de deformação do material em regiões de partículas de cromo e na matriz de ferro. Para determinar a distribuição de dureza foram realizadas 5 (cinco) identações em cada fase visível, caracterizadas por: uma fase clara (partículas de cromo) e uma fase em tom cinza (matriz de ferro), vide Figura 5. Os ensaios foram conduzidos com um microdurômetro equipado com um penetrador Vickers, empregando carga de 500gf e tempo de aplicação de 10s. A Figura 5 apresenta as identações aplicadas na mistura 1 (M1).

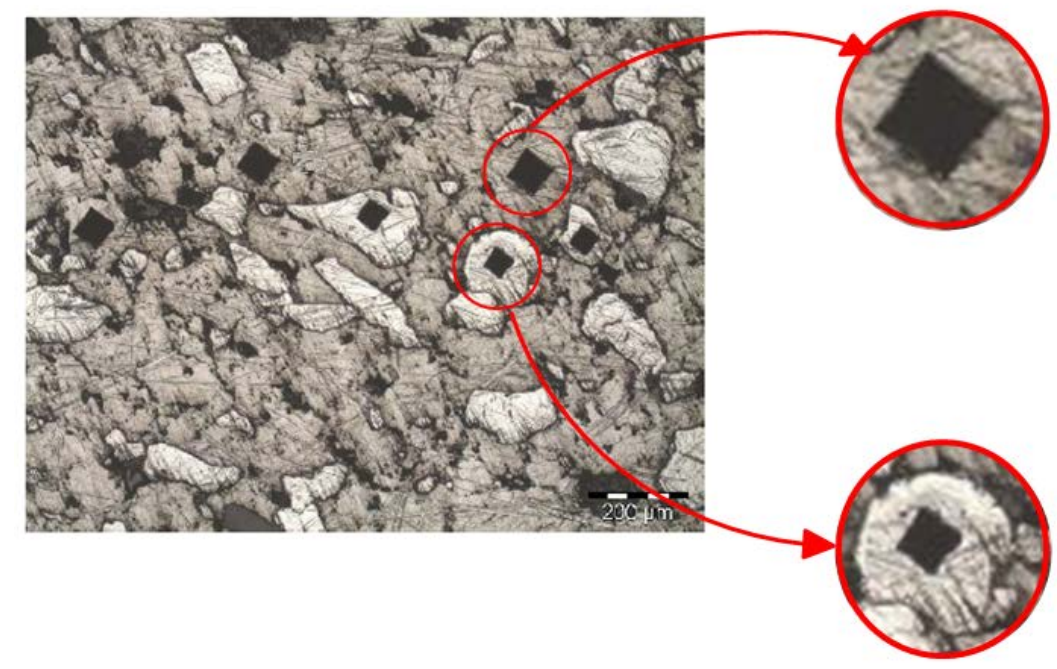

Figura 5: Imagem de MO de impressões do penetrador no ensaio de microdureza HV0,5 instrumentado na mistura 1 (M1).

A Tabela 4 apresenta os valores obtidos no ensaio, onde percebe-se valores de microdureza maiores para as partículas de cromo em comparação com a matriz de ferro.

Tabela 4: Resultados do ensaio de microdureza onde apresenta as duas fases presentes nas amostras, fase clara (cromo) e fase em tom cinza (matriz de ferro)

\begin{tabular}{c|c|c}
\hline \multirow{2}{*}{ Mistura } & $\begin{array}{c}\text { (Região) } \\
\text { Fase }\end{array}$ & Microdureza Média HV \\
\hline \multirow{2}{*}{ Mistura 1 } & $\mathrm{Cr}$ & $236 \pm 25$ \\
\cline { 2 - 3 } & $\mathrm{Fe}$ & $148 \pm 55$ \\
\hline \multirow{2}{*}{ Mistura 5 } & $\mathrm{Cr}$ & $351 \pm 31$ \\
\cline { 2 - 3 } & $\mathrm{Fe}$ & $137 \pm 16$ \\
\hline \multirow{2}{*}{ Mistura 8 } & $\mathrm{Cr}$ & $1001 \pm 89$ \\
\cline { 2 - 3 } & $\mathrm{Fe}$ & $220 \pm 33$ \\
\hline \multirow{2}{*}{ Mistura 10 } & $\mathrm{Cr}$ & $1110 \pm 85$ \\
\cline { 2 - 3 } & $\mathrm{Fe}$ & $379 \pm 38$ \\
\hline
\end{tabular}

Para uma melhor visualização dos resultados da Tabela 4, construiu-se um gráfico (Figura 6) mostrando o aumento da dureza conforme a elevação da temperatura de sinterização das formulações testadas. A partir destes resultados, se observa que a matriz apresentou valores mais elevados de dureza quando a temperatura de $1200^{\circ} \mathrm{C}$ foi utilizada para a sinterização. Este fenômeno pode ser explicado pela difusão do elemento de liga $\mathrm{Ni}$, que, uma vez difundido na matriz, ocasiona um aumento da resistência mecânica do material. Estes resultados estão de acordo com a literatura, sendo o Ni o elemento de liga que apresenta maior influência na resistência mecânica do material, associado a sua característica de facilitar a formação da fase austenita [14]. Adicionalmente, os resultados obtidos neste ensaio de microdureza indicam que a dureza das partículas de cromo também aumenta consideravelmente quando a temperatura de sinterização atinge o valor de $1200^{\circ} \mathrm{C}$ (amostras M8 e M10). 
Os resultados obtidos para dureza HV estão de acordo com a literatura [15]o qual indica que o Cr não é o elemento responsável pelo endurecimento do aço, o cromo é adicionado, principalmente com a função de formar a camada passivadora comum aos aços inoxidáveis [14].

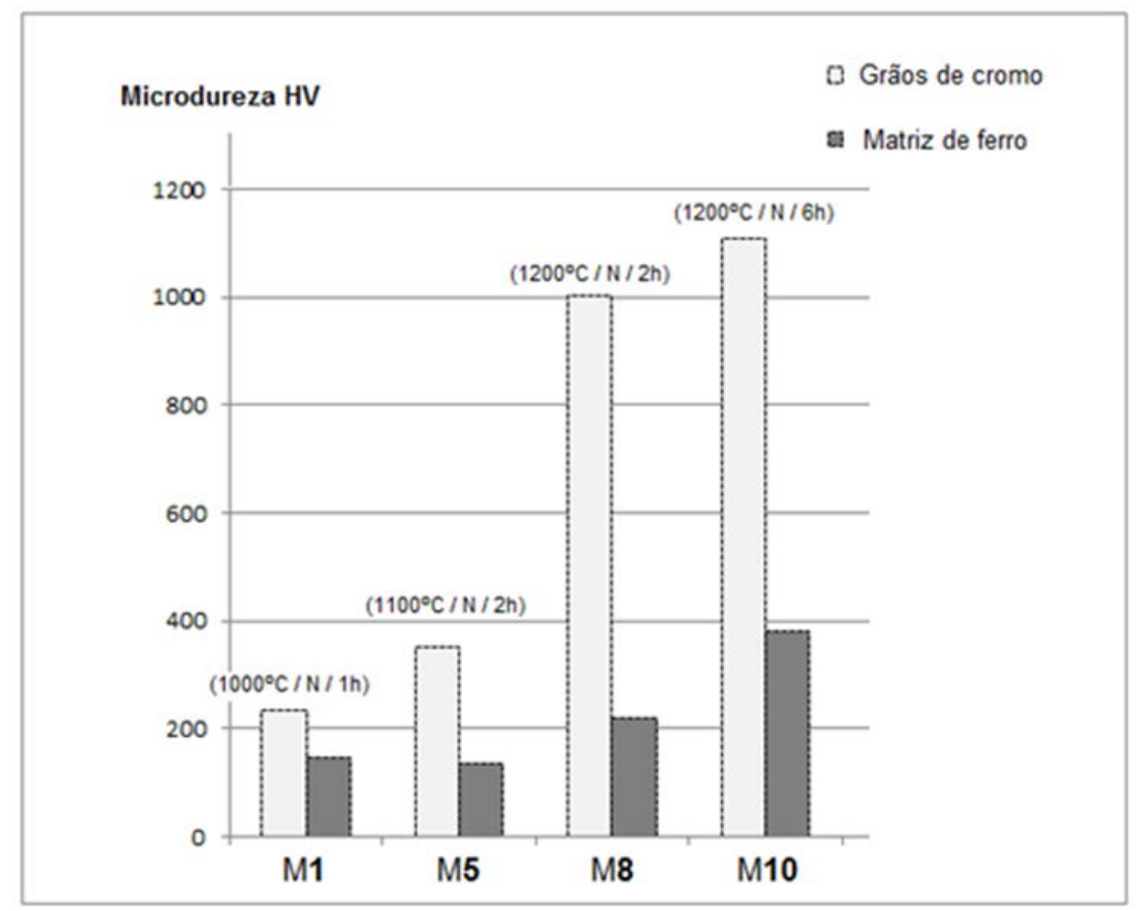

Figura 6: Gráfico com os resultados de microdureza das misturas M1, M5, M8 e M10

Comparando os resultados obtidos com a literatura, segundo Outokumpu [15], a dureza média para o aço inoxidável duplex UNS S32205 é 235 HV. As misturas M1, M5, M8 apresentaram valores superiores a 235 HV nas regiões de grãos de cromo e inferiores na matriz de ferro. Já a mistura M10 apresentou valores superiores nas regiões de partículas de cromo e na matriz de ferro.

\section{CONCLUSÕES}

Este trabalho tratou da produção de uma liga obtida por sinterização metálica pela mistura de pós elementares dissociados de Fe-Cr-Ni-Mo-Mn, além de avaliar a difusividade dos elementos $\mathrm{Cr}$ e Ni na matriz de ferro, e apresentar os valores de dureza obtidos na liga.

Com a análise de DRX verificou-se picos relativos à presença das fases de austenita e ferrita. O parâmetro comercialmente utilizado para obter as melhores propriedades mecânicas, resistência à corrosão e elevada tenacidade da liga, é quando a mesma apresentar um balanceamento próximo de $50 \%$ ferrita e $50 \%$ austenita. No entanto, no presente trabalho a austenita apresentou picos com baixa intensidade evidenciando baixo percentual da mesma. Observou-se, ainda, um deslocamento dos picos da austenita inerentes ao processo de compactação que deformou as partículas e acarretou neste pequeno deslocamento para a esquerda, mas sobretudo, pela baixa difusão do cromo nas partículas de ferro, o que impediu a homogeneização e formação da liga duplex.

As análises de MEV e (EDS), apresentaram a difusão do Ni e Cr na liga de Fe. Foi possível verificar que o Ni que difundiu na matriz de ferro e em menor proporção nas partículas de cromo, em todos os parâmetros avaliados. Já o Cr não apresentou boa difusão na matriz de ferro.

Para os ensaios de microdureza se notou que elevando à isoterma e o tempo das formulações no forno, o valor aumenta, sendo superior em todos os parâmetros nas partículas de cromo. Já na mistura 10 se observou que a matriz de ferro apresentou valores de microdureza superiores aos apresentados pela literatura, podendo ter relação com a formação de nitretos ocasionadas pelo tempo de sinterização de 360 minutos a $1200^{\circ} \mathrm{C}$.

Por fim, pôde se observar que as partículas de cromo sofreram oxidação na sua superfície, o que impediu que as mesmas pudessem difundir na matriz de ferro. No entanto, a baixa difusão de Cr na matriz de ferro não influenciou suas propriedades mecânicas, tendo em vista que o elemento de maior influência nestas propriedades é o Ni, que apresentou boa difusividade. 


\section{AGRADECIMENTOS}

Os autores agradecem a FAPERGS (Fundação de Amparo à Pesquisa do Estado do Rio Grande do Sul) pelo apoio financeiro.

\section{BIBLIOGRAFIA}

[1] YONEKUBO, A. E. Caracterização Microestrutural do aço inoxidável superduplex UNS S 32520 (UR $52 \mathrm{~N}^{+}$) processado por moagem de alta energia. Dissertação de M.Sc., UEPG, Ponta Grossa, Brasil, 2010.

[2] MARTINS, M. Caracterização microestrutural-mecânica e resistência à corrosão do aço inoxidável super duplex ASTM A890 / A890M Grau 6ª Tese de D.Sc.,Universidade de São Paulo, São Paulo, SP, Brasil, 2006.

[3] SOUZA JUNIOR, C. M. Influência da Deformação e do Tratamento Térmico na Textura Cristalográfica de um Aço Inoxidável Duplex, Tese de D.Sc., COPPE/UFRJ, Rio de Janeiro, RJ, Brasil 2008.

[4] MOINHOS, C. Obtenção do aço inoxidável duplex Fe-19,5Cr-5Ni utilizando moagem de alta energia, Dissertação M.Sc., UEPG, Ponta Grossa, Brasil, 2005.

[5] DOBRZANSKI, L. A., BRYTAN, Z., GRANDE, M., et al. Properties of Duplex Stainless steels made by Powder Metallurgy, Archives of Materials Science and Engineering, n.28, v.4, 217-223, 2007.

[6] GARCÍA, C., MARTIN, F., BLANCO, Y. "Effect of sintering cooling rate on corrosion resistance of powder metallurgy austenitic, ferritic and duplex stainless steels sintered in nitrogen”, Corrosion Science n.61, pp.45-52, 2012.

[7] MARTIN, F. GARCÍA, C. BLANCO, Y., et al., "Tribocorrosion behaviour of powder metallurgy duplex stainless steels sintered in nitrogen”, Tribology International , n.57, pp.76-85, 2013.

[8] TANSKI, T., BRYTAN, Z., LABISZ, K. “Fatigue behaviour of sintered duplex stainless steel”, In:XVII International Colloquium on Mechanical Fatigue of Metals (ICMFM17), Procedia Engineering, n.74, pp.421 - 428, 2014.

[9] AMERICAN SOCIETY FOR TESTING AND MATERIALS. ASTM A 240/A 240M-99 - "Standard Specification for Chromium and Chromium-Nickel Stainless Steel Plate, Sheet, and Strip for Pressure Vessels and for General Applications”, ASTM International, West Conshohocken, PA, 2004.

[10] SPOMBERG, S. Uso de Difratometria de Raios-X na Quantificação de Ferrita e Austenita em Aços Inoxidáveis Dúplex, Tese de D.Sc., FEI, São Bernardo do Campo, SP, Brasil, 2010.

[11] ICDD- “International Centre for Diffraction Data”, 2004.

[12] DOWNS, R.T. The RRUFF Project: na integrad study of the chemistry, crystallography, Raman na infrared spectroscopy of minerals, In: Program and Abstracts of the 19th General Meeting of the Internacional Mineralogical Association, Kobe, Japan, pp.003-13, 2006.

[13] BIEHL, L.V., 2002, Estudo da Carburização de Tubos de Ligas de Fe-Cr-Ni Aplicadas em Fornos de Pirólise, Tese de D.Sc., PPGEM / UFRGS, Rio Grande do Sul, RS, Brasil.

[14] CHIAVERINI, V. Aços e ferros fundidos, São Paulo, ABM(Associação Brasileira de Metais), 1990.

[15] OUTOKUMPU. “Handbook of Stainless Steel”, Outokumpu Oyj, 2013. 